\title{
Finite-volume effects and the electromagnetic contributions to kaon and pion masses
}

\section{S. Basak ${ }^{a}$, A. Bazavov ${ }^{b}$, C. Bernard ${ }^{* c}$, C. DeTar ${ }^{d}$, E. Freeland ${ }^{e}$, J. Foley $^{d}$,} Steven Gottlieb ${ }^{f}$, U.M. Heller ${ }^{g}$, J. Komijani ${ }^{c}$, J. Laiho $^{h}$, L. Levkova ${ }^{d}$, J. Osborn ${ }^{i}$, R.L. Sugar ${ }^{j}$, A. Torok ${ }^{f \dagger}$, D. Toussaint ${ }^{k}$, R.S. Van de Water ${ }^{l}$, R. Zhou ${ }^{l}$

${ }^{a}$ NISER, Bhubaneswar, Orissa 751005, India

${ }^{b}$ Department of Physics and Astronomy, University of Iowa, Iowa City, IA 52240, USA

${ }^{c}$ Department of Physics, Washington University, St. Louis, MO 63130, USA

${ }^{d}$ Department of Physics and Astronomy, University of Utah, Salt Lake City, UT 84112, USA

${ }^{e}$ Liberal Arts Department, School of the Art Institute of Chicago, Chicago, IL, USA

${ }^{f}$ Department of Physics, Indiana University, Bloomington, IN 47405, USA

${ }^{g}$ American Physical Society, One Research Road, Ridge, NY 11961, USA

${ }^{h}$ Department of Physics, Syracuse University, Syracuse, NY 13244, USA

${ }^{i}$ ALCF, Argonne National Laboratory, Argonne, IL 60439, USA

${ }^{j}$ Physics Department, University of California, Santa Barbara, CA 93106, USA

${ }^{k}$ Physics Department, University of Arizona Tucson, AZ 85721, USA

${ }^{l}$ Theoretical Physics Department, Fermi National Accelerator Laboratory, Batavia 60510, USA

\section{MILC Collaboration}

E-mail: cbewust 1 . edu

\begin{abstract}
We report on the MILC Collaboration calculation of electromagnetic effects on light pseudoscalar mesons. The simulations employ asqtad staggered dynamical quarks in QCD plus quenched photons, with lattice spacings varying from 0.12 to $0.06 \mathrm{fm}$. Finite volume corrections for the MILC realization of lattice electrodynamics have been calculated in chiral perturbation theory and applied to the lattice data. These corrections differ from those calculated by Hayakawa and Uno because our treatment of zero modes differs from theirs. Updated results for the corrections to "Dashen's theorem" are presented.
\end{abstract}

The 32nd International Symposium on Lattice Field Theory

23-28 June, 2014

Columbia University, New York, $N Y$

\footnotetext{
* Speaker.

${ }^{\dagger}$ current address: Intel Corporation, Hillsboro, OR 97124, USA
} 
Introduction. - The up and down quark masses may be calculated on the lattice from the masses of the $K^{+}$and $K^{0}$. To do so, one must remove the effects of electromagnetism (EM) from the kaon system. In lattice determinations of the phenomenologically important quantity $m_{u} / m_{d}$, both by us [1,2] and by other groups [3], the uncertainty in the EM contributions to kaon masses is the largest source of error. We have been working on reducing these uncertainties by calculating the EM effects directly on the lattice; progress has been reported previously in Refs. [4, 5].

Electromagnetism affects the calculation of $m_{u} / m_{d}$ from kaon masses primarily through the mass splitting $\left(M_{K^{ \pm}}^{2}-M_{K^{0}}^{2}\right)^{\gamma}$, where $\gamma$ denotes the EM contribution, i.e., the difference between this quantity in the real world and in a world where all quark charges are set to zero (keeping renormalized quark masses unchanged). Dashen's theorem [6] states that this kaon mass splitting is equal to the pion splitting $\left(M_{\pi^{ \pm}}^{2}-M_{\pi^{0}}^{2}\right)^{\gamma}$ at lowest order (LO) in chiral perturbation theory $(\chi \mathrm{PT})$. However, the corrections to the LO relation are not small. One may parameterize them by [3]

$$
\left(M_{K^{ \pm}}^{2}-M_{K^{0}}^{2}\right)^{\gamma}=(1+\varepsilon)\left(M_{\pi^{ \pm}}^{2}-M_{\pi^{0}}^{2}\right)^{\operatorname{expt}},
$$

where the experimental pion splitting is used, rather than the pion EM splitting. The two are equal up to isospin violating effects, which are NNLO in the pion system and therefore small.

Here, we describe our calculation of $\varepsilon$ on the lattice, using unquenched QCD but quenched photons. The EM-quenching effects on $\varepsilon$ may be calculated and corrected to NLO in SU(3) $\chi \mathrm{PT}$, with controlled errors [7]. We calculate $\varepsilon$ by computing $\left(M_{K^{ \pm}}^{2}-M_{K^{0}}^{2}\right)^{\gamma}$, and either using $\left(M_{\pi^{ \pm}}^{2}-\right.$ $\left.M_{\pi^{0}}^{2}\right)^{\text {expt }}$ or $\left(M_{\pi^{ \pm}}^{2}-M_{\pi^{0}}^{2}\right)^{\gamma}$ in the right-hand side of Eq. (1). Determining the mass of the true $\pi^{0}$ is costly, however, since it has disconnected EM diagrams even in the isospin limit. Instead, we drop the disconnected diagrams, which are expected to be small, and simply find the RMS average mass of $u \bar{u}$ and $d \bar{d}$ mesons. We call the pion obtained in this manner the " $\pi^{0}$." Both the true $\left(M_{\pi^{0}}^{2}\right)^{\gamma}$ and our $\left(M_{“ \pi^{0},}^{2}\right)^{\gamma}$ are small since EM contributions to neutral mesons vanish in the chiral limit.

A key unresolved issue in our previous work was the size of the finite volume (FV) effects. Comparisons of results on two lattice volumes showed unexpectedly small differences [5], making it difficult to determine the true size of the effect. Here, we examine the FV effects in more detail, and show that they can be predicted with good accuracy in one-loop (staggered) chiral perturbation theory. We can therefore correct for FV effects, with a small residual systematic error. After doing so we are able to quote a new result for the parameter $\varepsilon$, and give corresponding results for $m_{u} / m_{d}$.

Lattice setup. - We calculate meson masses on the (2+1)-flavor MILC asqtad ensembles, with quenched photon fields, and with lattice spacings ranging from $\approx 0.12 \mathrm{fm}$ to $\approx 0.06 \mathrm{fm}$. Table 1 shows the ensembles employed. The valence quarks have charges $\pm 2 / 3 e, \pm 1 / 3 e$ or 0 , where $e=e_{\text {phys }}$ or (on most ensembles) $2 e_{\text {phys }}$ or $3 e_{\text {phys }}$, with $e_{\text {phys }} \approx 0.303$ the physical electron charge.

QED in Finite Volume. - With the non-compact realization of QED on the lattice, which we use, it is necessary to drop some zero-modes in a finite volume in order to have a convergent path integral. In particular, the action in Coulomb gauge for the zero component of the vector potential, $A_{0}$, is $\frac{1}{2} \int\left(\partial_{i} A_{0}\right)^{2}$. Since the $A_{0}$ mode with spatial momentum $\vec{k}=0$ has vanishing action, it must be dropped. Similarly, the action for the spatial components $A_{i}$ is $\frac{1}{2} \int\left[\left(\partial_{0} A_{i}\right)^{2}+\left(\partial_{j} A_{i}\right)^{2}\right]$. Here only the mode with 4-momentum $k_{\mu}=0$ must be dropped, and that is what we do. Hayakawa and Uno, in their calculation of EM FV effects in $\chi \mathrm{PT}[8]$, drop all $A_{i}$ modes with $\vec{k}=0$. This means that the FV effects in the MILC calculations are different from those computed in Ref. [8]. 


\begin{tabular}{|c|c|c|c|c|c|c|}
\hline \hline$\approx a[\mathrm{fm}]$ & Volume & $\beta$ & $m_{l}^{\prime} / m_{s}^{\prime}$ & \# configs. & $L(\mathrm{fm})$ & $m_{\pi} L$ \\
\hline \hline 0.12 & $12^{3} \times 64^{*}$ & 6.76 & $0.01 / 0.05$ & 1000 & 1.4 & 2.7 \\
& $16^{3} \times 64^{*}$ & 6.76 & $0.01 / 0.05$ & 1303 & 1.8 & 3.6 \\
& $20^{3} \times 64$ & 6.76 & $0.01 / 0.05$ & 2254 & 2.3 & 4.5 \\
& $28^{3} \times 64$ & 6.76 & $0.01 / 0.05$ & 274 & 3.2 & 6.3 \\
& $40^{3} \times 64^{*}$ & 6.76 & $0.01 / 0.05$ & 115 & 4.6 & 9.0 \\
& $48^{3} \times 64^{*}$ & 6.76 & $0.01 / 0.05$ & $132+52$ & 5.4 & 10.8 \\
& $20^{3} \times 64$ & 6.76 & $0.007 / 0.05$ & 1261 & 2.3 & 3.8 \\
& $24^{3} \times 64$ & 6.76 & $0.005 / 0.05$ & 2099 & 2.7 & 3.8 \\
\hline 0.09 & $28^{3} \times 96$ & 7.09 & $0.0062 / 0.031$ & 1930 & 2.3 & 4.1 \\
& $40^{3} \times 96$ & 7.08 & $0.0031 / 0.031$ & 1015 & 3.3 & 4.2 \\
\hline 0.06 & $48^{3} \times 144$ & 7.47 & $0.0036 / 0.018$ & 670 & 2.8 & 4.5 \\
\hline \hline
\end{tabular}

Table 1: Parameters of the (2+1)-flavor asqtad ensembles used in this study. Volumes marked with $*$ are currently used in the FV studies but not in the full analysis. The quark masses $m_{l}^{\prime}$ and $m_{s}^{\prime}$ are the light and strange dynamical masses used in the runs. The number of configurations listed as ' $132+52$ ' for the $a \approx 0.12 \mathrm{fm}, 48^{3} \times 64$ ensemble gives values for two independent streams, the first in single precision, and the second in double. At the moment, we treat them as separate data, and do not average the results. The $40^{3} \times 64$ and $48^{3} \times 64 a \approx 0.12 \mathrm{fm}$ ensembles are new since the conference and are still being analyzed.

To make explicit the difference between our set-up and that of Ref. [8], we give the spatial components of the photon propagator in each case:

$$
\begin{aligned}
& \left\langle A_{i}(k) A_{j}(-k)\right\rangle= \begin{cases}\frac{1}{k^{2}}\left(\delta_{i j}-\frac{k_{i} k_{j}}{\vec{k}^{2}}\right), & \vec{k} \neq 0 ; \\
0, & \vec{k}=0 .\end{cases} \\
& \left\langle A_{i}(k) A_{j}(-k)\right\rangle= \begin{cases}\frac{1}{k^{2}}\left(\delta_{i j}-\frac{k_{i} k_{j}}{\vec{k}^{2}}\right), & \vec{k} \neq 0 ; \\
\frac{1}{k^{2}} \delta_{i j}, & \vec{k}=0, k_{0} \neq 0 ; \\
0, & \vec{k}=0, k_{0}=0 .\end{cases}
\end{aligned}
$$

The propagator of the time component $A_{0}$ is the same in both cases, and is simply $1 / \vec{k}^{2}$ for $\vec{k} \neq 0$, and 0 for $\vec{k}=0$. The violation of Gauss's Law induced by the absence of the $\vec{k}=0 A_{0}$ mode makes it possible to have net charges on a FV torus with periodic boundary conditions [8]. But Gauss's Law has no implications for the spatial modes $A_{i}$, so does not distinguish between Eqs. (2) and (3).

We note that the BMW Collaboration [9] has recently calculated the FV effects for the QED in Eqs. (2) and (3), which they call $\mathrm{QED}_{L}$ and $\mathrm{QED}_{T L}$, respectively. As part of their study of the proton-neutron mass difference in dynamical QCD+QED, they have independently found the key FV results described below, and have also worked out explicit asymptotic forms in large volumes.

Finite Volume Effects in Chiral Perturbation Theory. - The EM effects on pseudoscalar meson masses have been calculated to NLO in staggered chiral perturbation theory (S $\chi$ PT) [10]. The calculation gives the splitting in squared mass, $\Delta M_{x y}^{2} \equiv M_{x y}^{2}-M_{x^{\prime} y^{\prime}}^{2}$, between a Goldstone (taste $\xi_{5}$ ) meson made from valence quarks $x$ and $y$ with charges $q_{x}, q_{y}$ and masses $m_{x}, m_{y}$, and the corresponding meson made from quarks $x^{\prime}$ and $y^{\prime}$ with the same masses but the quark charges set to zero. The explicit formula for $\Delta M_{x y}^{2}$ in terms of NLO chiral logarithms and low-energy constants (LECs) can be found in Refs. [5, 10].

The logarithms in the chiral expressions come from three Feynman diagrams. Two of them, the sunset graph and the photon tadpole, have internal low-energy photons, while one, the meson 
tadpole, has only an internal meson line. The FV effects coming from the meson tadpole are completely standard (see Ref. [11] for the explicit formulas we use), and are in fact tiny for all our ensembles. On the other hand, one should expect large FV effects from the photon graphs, even on the largest of our ensembles, since the photon is massless.

Although the photon tadpole diagram vanishes in dimensional regularization in infinite volume (IV), since there are no dimensional parameters in the integral, it is important in FV. In fact, we need to add the tadpole to the sunset diagram in order for the FV difference (FV result minus IV result) to be finite in Coulomb gauge. Once the photon diagrams are added, we can simply perform a brute-force computation of the FV difference. We do this using the importance-sampling integration program VEGAS [12]. The VEGAS integrand is taken to be the difference between the IV integrand, and the distance-weighted average of its evaluations at the 16 corners of the FV hypercube containing the point. We have checked that our result for the sum of the sunset and the photon tadpole diagrams agrees with that of Ref. [8] when Eq. (2) is used.

The difference between the Hayakawa and Uno result and the MILC result is simply due to the modes $\vec{k}=0, k_{0} \neq 0$ in Eq. (3). This difference can easily be worked out analytically, and is $q T / 4 L^{3}$, where $q$ is the meson charge, and $T$ and $L$ are the time and spatial length of the lattice, respectively. Note that this gives a rather subtle large-volume behavior in the MILC case: The result is acceptable if the limit $L \rightarrow \infty$ is taken before $T \rightarrow \infty$, or if the limits are taken together at fixed aspect ratio $T / L$, but not if the limit $T \rightarrow \infty$ is taken first. In other words, the MILC set-up is not well defined in finite spatial volume at zero temperature. This fact has also been pointed out by BMW [9]. They make the further point that the $\mathrm{QED}_{T L}$ (MILC) set-up violates reflection positivity because the constraint required to set the single $k_{\mu}=0$ mode of $A_{i}$ to zero involves the square of the integral over all space-time of $A_{i}$. Although many actions used in lattice QCD violate reflection positivity, one might worry that in this case the violation leads to problems with defining or isolating the lowest states in correlation functions. In practice, this does not seem to be a problem for us. We find no discernible differences between the qualities of plateaus in correlation functions in our QCD+quenched QED simulations versus those for QCD alone.

In Fig. 1 we show fits to the FV form derived in S $\chi$ PT for two different meson masses on the $a \approx 0.12 \mathrm{fm}$ ensembles with $a m_{l}^{\prime}=0.01, a m_{s}^{\prime}=0.05$. The shape of the fit curves are completely determined in NLO S $\chi$ PT; the only free parameter in each fit is the overall height of the curve. The theory at NLO gives a reasonably good description of the data, and we use it to correct the data for FV effects. To estimate the systematic error associated with this correction (a 'residual' FV error), we examine the deviations of the fit lines from the data in Fig. 1. By far the largest deviation occurs for the 'pion' (blue) curve at $L=16$. Although the fit there misses the point by $\approx 4 \sigma$, the difference between the data and the IV value is $70 \%$ of the FV correction implied by the fit. Below, we therefore take the residual FV error to be $30 \%$ of the correction. Since the deviation at all other points in Fig. 1 is much less than 30\%, we believe this error estimate to be conservative. Note that a correction from higher order terms in $\chi \mathrm{PT}$ of $\sim 30 \%$ is somewhat large, but not unreasonable.

One can now understand why it was difficult to observe FV effects directly in the data set available in Ref. [5]. At that time, we had only the $L=20$ and $L=28$ ensembles to compare. From Fig. 1, one sees that the minima of the curves are in this region of $L$ or close to it, and therefore the difference expected between these volumes is small compared to the statistical errors in the data.

Chiral Fits. - Once FV effects have been removed, we fit the data to the IV NLO S $\chi$ PT form, 


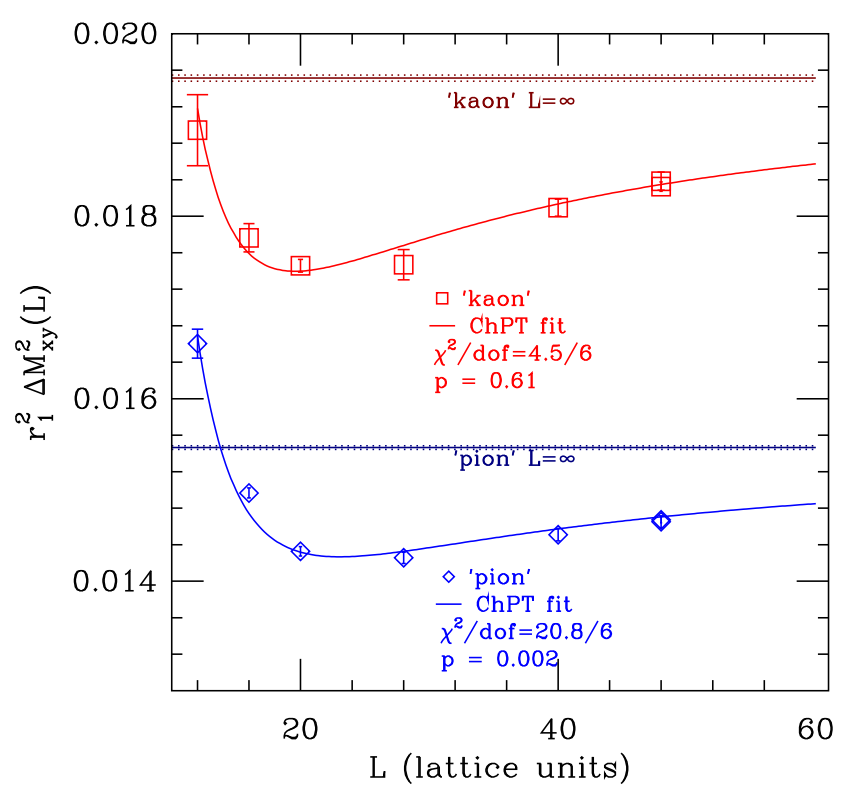

Figure 1: Finite volume effects at $a \approx 0.12 \mathrm{fm}$ and $a m_{l}^{\prime}=0.01, a m_{s}^{\prime}=0.05$ as a function of spatial lattice length $L$ for two different meson masses: a unitary 'pion' (blue) with degenerate valence masses $m_{x}=m_{y}=$ $m_{l}^{\prime}$, and a 'kaon' (red) with valence masses $m_{x}=m_{l}^{\prime}$ and $a m_{y}=0.04$, close to the physical strange quark mass. The fit lines are to the FV form from $\mathrm{S} \chi \mathrm{PT}$, and have one free parameter each, the infinite volume value (shown by horizontal solid lines with dotted lines for errors).

plus NNLO analytic terms, as described in [5]. The higher order analytic terms, which include discretization terms, are necessary because our statistical errors in $\Delta M_{x y}^{2}$ are $\sim 0.3 \%$ for charged mesons and $\sim 1.0 \%$ for neutral mesons. When we include data with charges greater than physical, analytical terms of order $\alpha_{E M}^{2}$ are also necessary to obtain acceptable fits.

Figure 2 shows our data after correction for FV effects, along with the chiral fit and its extrapolations. The FV corrections are roughly $7-10 \%$ for 'pions' (shown on the left) and 10-18\% for 'kaons' (shown on the right). The corrections are larger at higher mass because of the overall factor of $M^{2}$ in the one-loop logarithms that give the FV effects, but not in the LO term, which is mass independent by Dashen's theorem. Partially quenched charged- and neutral-meson data, with $e= \pm e_{\text {phys }}$ and $e= \pm 2 e_{\text {phys }}$, are fit simultaneously, but only data for unitary or approximately unitary mesons with charge $\pm e_{\text {phys }}$ is shown in Fig. 2. This fit has 149 data points and 29 parameters, with $\chi^{2}=127$, for an (uncorrelated) $p=0.34$.

With our current data set, correlated fits generally give very low $p$ values. Even after drastic thinning of the data to reduce the number of small eigenvalues of the correlation matrix, correlated fits achieve, at best, $p \sim 0.01$. The central fit shown in Fig. 2 is therefore an uncorrelated fit, although we do include the correlated fits as alternatives in the estimate of the systematic error. We note that we have some preliminary data on additional ensembles with $a \approx 0.06 \mathrm{fm}$ and $a \approx 0.045$ $\mathrm{fm}$, not shown in Table 1 . With that data, we may drop the coarsest ( $a \approx 0.12 \mathrm{fm}$ ) ensembles from the fit. Good correlated fits then become possible, even without extreme thinning of the data.

The black lines in Fig. 2 show the fit after setting valence and sea masses equal, adjusting $m_{s}$ to its physical value, extrapolating to the continuum, and adjusting the sea charges to their physical values using NLO $\chi$ PT. The last adjustment vanishes identically for pions and is very small for kaons. From the black lines for the $\pi^{+}$and $K^{+}$, we subtract the corresponding results for the 


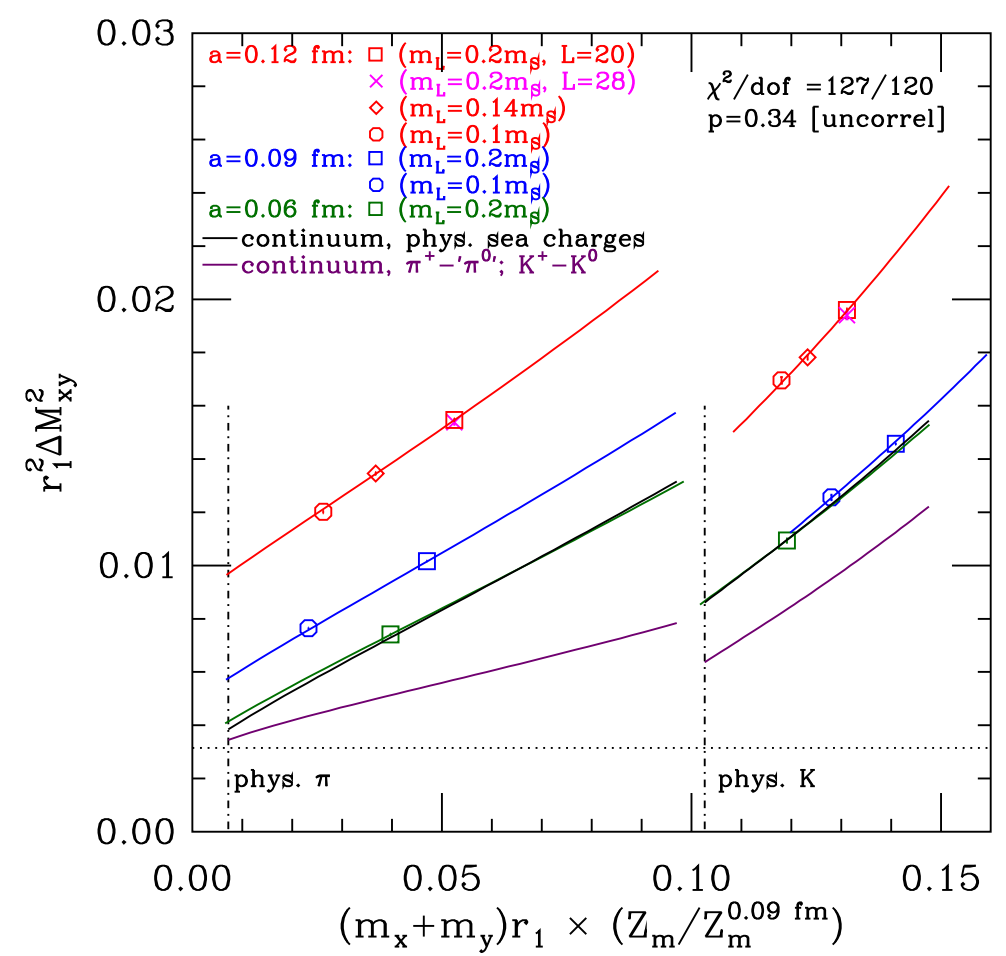

Figure 2: Central fit to the EM splitting $\Delta M_{x y}^{2} v s$. the sum of the valence-quark masses. Only a small subset of the partially quenched data set included in the fit is shown: the points for $a \approx 0.09 \mathrm{fm}$ and $\approx 0.06 \mathrm{fm}$, as well as the 'pion' (left hand) points for $a \approx 0.12 \mathrm{fm}$, have unitary values of the valence masses, while the 'kaon' (right hand) points for $a \approx 0.12 \mathrm{fm}$ have $m_{x}=m_{l}^{\prime}$ but $m_{y}=0.8 m_{s}^{\prime}$, which is closer to the physical strange mass than $m_{s}^{\prime}$ itself. All points shown are for mesons with charge $\pm e_{\text {phys }}$; neutral mesons and mesons with charges $\pm 2 e_{\text {phys }}$ are also included in the fit. The data have been corrected for FV effects using NLO $\mathrm{S} \chi \mathrm{PT}$. The red, blue and green curves correspond to the three lattice spacings. The black and purple curves are extrapolations, see text. The horizontal dotted line is the experimental value of the $\pi^{+}-\pi^{0}$ splitting.

neutral mesons, " $\pi^{0}$ " and $K^{0}$, giving the purple lines, whose values at the physical point for each meson (shown with the vertical dashed-dotted lines) give the physical results.

Errors, Results, and Outlook. - From the central fit, and using $\left(M_{\pi^{ \pm}}^{2}-M_{\pi^{0}}^{2}\right)^{\gamma}$ in the right-hand side of Eq. (1), we find $\varepsilon=0.84(5)$. Without correction for FV effects, we found $\varepsilon=0.65(7)$ [5]. We take 0.06 , which is $30 \%$ of the size of the correction, as the residual FV error. One way to estimate other lattice systematic errors is to consider a wide variety of alternative chiral/continuum fits: different amounts of thinning of the data, different cutoffs on the highest values of considered, removal of various NNLO chiral and discretization terms, imposition of power-counting priors on the NNLO or NLO terms, and whether or not to include correlations. While most of these alternatives give results fairly close to the central value, there are some outliers that also have fairly large statistical errors, especially among the uncorrelated fits. Weighting the results by the inverse square of their statistical errors, the standard deviation of $\varepsilon$ over all these alternatives is 0.07 . The unweighted standard deviation is 0.13 , and largest amount by which differences from the central value exceed the statistical error is 0.21 . Since these possible estimates of the error differ greatly, we choose instead to make the estimate by simply replacing $\left(M_{\pi^{ \pm}}^{2}-M_{\pi^{0}}^{2}\right)^{\text {expt }}$ instead of $\left(M_{\pi^{ \pm}}^{2}-M_{\pi^{0}}^{2}\right)^{\gamma}$; if lattice errors were absent these two results would agree to good accuracy. This gives a difference of 0.18 , from the central value, close to the largest of the other estimates. We 
thus find the following preliminary result:

$$
\varepsilon=0.84(5)_{\text {stat }}(18)_{a^{2}}(6)_{\mathrm{FV}} .
$$

Using this value for $\varepsilon$, our estimate for the EM uncertainty in $m_{u} / m_{d}$ is reduced by more than a factor of 2 from our error in Ref. [1]. Using the MILC HISQ (2+1+1)-flavor QCD ensembles, as reported in Ref. [2], we obtain, preliminarily,

$$
m_{u} / m_{d}=0.4482(48)_{\mathrm{stat}}\left({ }_{-115}^{+21}\right)_{a^{2}}(1)_{\mathrm{FV}_{\mathrm{QCD}}}(165)_{\mathrm{EM}},
$$

where here "EM" denotes all errors from $\mathrm{EM}$, while "FV $\mathrm{QCD}$ " refers to finite-volume effects in the pure QCD calculation. We are currently finishing the analysis on the additional ensembles at $a \approx 0.06 \mathrm{fm}$ and $a \approx 0.045 \mathrm{fm}$. Preliminary indications are that the lattice errors in $\varepsilon$ will be significantly reduced by the inclusion of these ensembles.

Acknowledgments: We thank Laurent Lellouch, Antonin Portelli, and Francesco Sanfilippo for useful discussions. The spectrum running was done on computers at the National Center for Supercomputing Applications, Indiana University, the Texas Advanced Computing Center (TACC), and the National Institute for Computational Science (NICS). Configurations were generated with resources provided by the USQCD Collaboration, the Argonne Leadership Computing Facility, and the National Energy Research Scientific Computing Center, which are funded by the Office of Science of the U.S. Department of Energy; and with resources provided by the National Center for Atmospheric Research, NICS, the Pittsburgh Supercomputer Center, the San Diego Supercomputer Center, and TACC, which are funded through the National Science Foundation's XSEDE Program. This work was supported in part by the U.S. Department of Energy and the National Science Foundation. Fermilab is operated by Fermi Research Alliance, LLC, under Contract No. DEAC02-07CH11359 with the U.S. Department of Energy.

\section{References}

[1] A. Bazavov et al., Rev. Mod. Phys. 82, 1349 (2010) [arXiv:0903.3598]; A. Bazavov et al. [MILC], PoS LATTICE 2009, 079 (2009) [arXiv:0910.3618]; C. Aubin et al., [MILC], Phys. Rev. D 70, 114501 (2004) [hep-lat/0407028].

[2] A. Bazavov et al. [Fermilab Lattice and MILC], arXiv:1407.3772 [hep-lat].

[3] S. Aoki et al. [FLAG], arXiv:1310.8555 [hep-lat].

[4] S. Basak et al. [MILC], PoS LATTICE 2008, 127 (2008) [arXiv:0812.4486]; A. Torok et al. [MILC] PoS LATTICE 2010, 127 (2010).

[5] S. Basak et al. [MILC], PoS LATTICE 2012, 137 (2012) [arXiv:1210.8157] and PoS CD 12, 030 (2013) [arXiv:1301.7137 [hep-lat]].

[6] R. Dashen, Phys. Rev. 183, 1245 (1969).

[7] J. Bijnens and N. Danielsson, Phys. Rev. D 75, 014505 (2007) [hep-lat/0610127].

[8] M. Hayakawa and S. Uno, Prog. Theor. Phys. 120, 413 (2008) [arXiv:0804.2044 [hep-ph]].

[9] S. Borsanyi et al., arXiv:1406.4088 [hep-lat].

[10] C. Bernard and E. D. Freeland, PoS LATTICE 2010, 084 (2010) [arXiv:1011.3994].

[11] C. Bernard [MILC Collaboration], Phys. Rev. D 65, 054031 (2002) [hep-lat/0111051].

[12] G.P. Lepage, J. Comp. Phys. 27, 192 (1978). 